\title{
Cerebral vessel laminins and IFN- $\gamma$ define Trypanosoma brucei brucei penetration of the blood-brain barrier
}

\author{
Willias Masocha, ${ }^{1}$ Brita Robertson, ${ }^{1}$ Martin E. Rottenberg, ${ }^{2}$ Jama Mhlanga, ${ }^{1}$ \\ Lydia Sorokin, ${ }^{3}$ and Krister Kristensson ${ }^{1}$ \\ ${ }^{1}$ Department of Neuroscience and 2Microbiology and Tumour Biology Center, Karolinska Institutet, Stockholm, Sweden. \\ ${ }^{3}$ Experimental Pathology, Lund University, Lund, Sweden.
}

\begin{abstract}
Subspecies of Trypanosoma brucei cause severe brain diseases after penetration of the blood-brain barrier. We investigated whether cytokines that modulate inflammatory cell infiltration into the brain also influence $T$. brucei neuroinvasion. Migration of a rodent pathogenic $T$. brucei strain from the cerebral blood vessels into the brain parenchyma was impeded in IFN- $\gamma^{-/-}$, IFN- $\gamma$ receptor ${ }^{-/-}\left(\right.$IFN- $\left.\gamma \mathbf{R}^{-/-}\right)$, IL-12 $\mathbf{p} 40^{-/-}$, and recombinant activating gene-1-/- $\left(\mathrm{RAG}^{-1 /-}\right)$ mice as compared with their WT littermates despite higher levels of parasitemia in the mutant strains. Parasites accumulated in the perivascular compartment, confined between the endothelial and the parenchymal basement membranes, in certain areas of the brains of IFN- $\gamma^{-/-}$, IFN- $\gamma \mathbf{R}^{-/-}$, and $\mathrm{RAG}-1^{-/-}$mice. This accumulation occurred around endothelial basement membranes containing the laminin $\alpha 4$ chain, while blood vessels showing robust laminin $\alpha 5$ chain immunostaining were not associated with parasite infiltration. The number of $\mathrm{CD}^{+}$and $\mathrm{CD8}^{+} \mathrm{T}$ cells infiltrating the brain parenchyma was also reduced in the IFN- $\gamma^{-/-}$and IFN- $\gamma \mathbf{R}^{-/-}$mice. Our findings suggest that lymphocyte-derived IFN- $\gamma$ facilitates trypanosome penetration across cerebral blood vessels and that the site of penetration is determined by the composition of the basement membranes of these vessels.
\end{abstract}

\section{Introduction}

African trypanosomiasis is caused by several subspecies of the extracellular hemoflagellate Trypanosoma brucei. T. brucei parasites are transmitted by the tsetse fly, and after the initial skin infection, the parasites spread via the lymph and blood circulation within the host. The disease has been divided into an early hemolymphatic stage, in which the parasites are confined to the blood and lymph, and a late encephalitic stage, in which T. brucei and/or increased numbers of lymphocytes are found in the cerebrospinal fluid (reviewed in ref. 1). Human African trypanosomiasis, or sleeping sickness, caused by T. brucei rhodesiense has a time course lasting weeks to months, while the disease caused by T. brucei gambiense is more protracted. Both forms of the disease give rise to white matter encephalitis (leukencephalitis) and are fatal if left untreated. Arsenic compounds, which penetrate the blood-brain barrier (BBB) and can have severe and often fatal side effects, are still the drugs of choice for treatment of the encephalitic stage of the disease.

Investigating the mechanisms by which parasites penetrate the cerebral blood vessels and enter the brain parenchyma is, therefore, of fundamental importance for understanding the neuropathogenesis of African trypanosomiasis and for developing new treatment strategies. In experimental animals infected with the rodent pathogenic strain T. brucei brucei, parasites appear early during infection in the choroid plexus and other circumventricular

Nonstandard abbreviations used: BBB, blood-brain barrier; EAE, experimental autoimmune encephalomyelitis; GLUT-1, glucose transporter- 1 ; IFN- $\gamma$ R, IFN- $\gamma$ receptor; p.i., postinfection; RAG-1, recombinant activating gene-1; VSG, variant surface glycoprotein.

Conflict of interest: The authors have declared that no conflict of interest exists.

Citation for this article: J. Clin. Invest. 114:689-694 (2004)

doi:10.1172/JCI200422104 organs (2) that lack a BBB. At later stages, the parasites penetrate the $\mathrm{BBB}$ and enter the brain parenchyma, as revealed by double immunohistochemical labeling of parasites and brain endothelial cells in a rat model of the chronic disease (3). A larger number of parasites appear in the white matter and in the septal nuclei rather than in the cerebral neocortex.

Central nervous system vessels constitute a barrier to inflammatory cell infiltration, owing to both their integrated dynamic structure that comprises endothelial cells and astrocytes (4) and to their basement membranes (5). Two basement membranes can be identified in association with blood vessels in the brain: an endothelial basement membrane and a parenchymal basement membrane. The latter is produced principally by astrocytes and deposited at their endfeet, which delineate the border of the brain parenchyma (5). The major functional component of all basement membranes is the laminin family of glycoproteins, all members of which are heterotrimers composed of an $\alpha, \beta$, and $\gamma$ chain. To date, $5 \alpha, 4 \beta$, and $3 \gamma$ have been described; the laminin $\alpha$ chains carry the cell-binding domains and therefore represent the functionally active portion of each laminin (6-8). It has recently been shown in a murine experimental autoimmune encephalomyelitis (EAE) model that CNS endothelial basement membranes contain laminin $\alpha 4$ and laminin $\alpha 5$ chains, and that T cell transmigration occurs only at sites where laminin $\alpha 4$, but no laminin $\alpha 5$, chains exist (5). This suggests that laminin $\alpha 4$ is permissive for leukocyte transmigration, and that laminin $\alpha 5$ is inhibitory. These data also indicate that both the endothelial cell layer and its underlying basement membrane, and in particular the laminin isoforms contained within this basement membrane, play decisive roles in defining sites of leukocyte infiltration into the brain. The expression of the endothelial laminins is influenced by proinflammatory cytokines (5), such as IFN- $\gamma$, IL-12, and TNF- $\alpha$, 
which also regulate invasion of inflammatory cells into the brain parenchyma by modulating the expression of cellular adhesion molecules on endothelial cells or molecules involved in tight junction function, and by inducing chemokine release from cells in the brain (reviewed in refs. 9-11).

IFN- $\gamma$ is secreted by both the innate and the adaptive immune cells and when stimulated by IL-12 and IL-18 is essential for control of intracellular pathogens (12). Besides regulating expression of laminin isoforms, IFN- $\gamma$ induces different microbicidal effector mechanisms, enhances the expression of adhesion molecules and chemokines, and stimulates antigen presentation (12). IFN- $\gamma$ can also regulate the level of expression of other cytokines, such as IL-12, or cytokine receptors such as IL-12/IL-23 R $\beta 2$ (13) and IL-18R $\alpha$ (13-16). Cumulative studies have revealed that both the $B$ cell-mediated antibody responses and the Th1 cell responses leading to the production of IFN- $\gamma$ are required for maximum systemic host resistance to trypanosomes in mice, with IFN- $\gamma$ acting to induce macrophage trypanolytic and trypanostatic activities $(17,18)$. Paradoxically, IFN- $\gamma$ has been associated with enhanced susceptibility to T. brucei brucei and T. congolense infections, as it reportedly mediates immunosuppression (19-21) and increases T. brucei brucei proliferation (22).

Parasites infiltrating the brain parenchyma are likely to encounter the same cellular barriers and basement membranes encountered by leukocytes in an inflammation. That is, they first encounter the barrier consisting of the endothelium and its basement membrane, and then the parenchymal basement membrane and the associated astrocyte endfeet (5). Furthermore, T. brucei brucei infections are associated with $\mathrm{B}$ and $\mathrm{T}$ cell infiltrations. We have, therefore, investigated T. brucei brucei infiltration into the brain parenchyma with respect to the endothelial basement membrane laminins. We found that the presence of laminin $\alpha 4$, but not laminin $\alpha 5$, in the endothelial basement membrane is associated with T. brucei brucei passage through this membrane and that IFN- $\gamma$ facilitates transmigration of T. brucei brucei across the parenchymal basement membrane into the brain parenchyma. Specifically, passage of T. brucei brucei into the brain across the parenchymal basement membrane was impeded in the absence of IFN- $\gamma$ or mature $\mathrm{B}$ and $\mathrm{T}$ lymphocytes.

\section{Results}

In a first set of experiments, we compared parasitemia levels in IFN- $\gamma^{-/-}$, IFN- $\gamma$ receptor ${ }^{-/-}\left(\right.$IFN- $\left.\gamma \mathrm{R}^{-/-}\right)$, recombinant activating gene-1/- $\left(\mathrm{RAG}^{-1 /-}\right)$, and IL-12 $40^{-/-}$mice with those in WT mice. Increased parasitemia was recorded in IFN- $\gamma^{-/-}$, IFN- $\gamma \mathrm{R}^{-/-}$, and RAG-1//- mice, but not in IL-12p40-/- mice (Figure $1 \mathrm{~A}$ ).

Next we compared the distribution of T. brucei brucei in relation to vessels in brains from infected WT, IFN- $\gamma^{-/-}$, and IFN- $\gamma \mathrm{R}^{-/-}$mice. To assess whether parasites were located within blood vessels or in the brain parenchyma, we used double immunolabeling with antibodies to T. brucei brucei and glucose transporter-1 (GLUT-1), a marker for endothelial cells. Penetration of parasites into the stroma of the choroid plexus and circumventricular organs was observed 6 days postinfection (p.i.) in all groups of mice. Parasites were confined to the lumens of intracerebral vessels at this time point. Starting at day 13 p.i., the fraction of total parasites observed in the brain parenchyma versus that in the vessel lumens increased with time in WT mice (Figures $1 \mathrm{~B}$ and 2A). Parasites penetrated more readily into the parenchyma of the white matter and septal nuclei than they did into the cerebral cortex. There was no obvious gradient of parasite density from the ventricles, which contained the heavily parasite-infiltrated choroid plexus, to the parenchyma. Surprisingly, in IFN- $\gamma^{-/-}$mice, the majority of parasites were still located intravascularly at 20 days p.i. (Figure $2 \mathrm{~B}$ ). Moreover, in IFN- $\boldsymbol{\gamma}^{-/}$but not WT mice, we observed clusters of parasites around several cerebral blood vessels in the septal nuclei, hippocampi, and paraventricular thalamic nuclei. These blood vessels appeared to be postcapillary venules (Figure 2C). Perivascular aggregates of trypanosomes were not evident in the white matter, where the parasites were observed mainly inside the vessels. IFN- $\gamma-\mathrm{R}^{-/-}$mice also showed reduced T. brucei brucei penetration into the brain parenchyma, as well as marked perivascular cuffing of parasites, confirming a role for IFN- $\gamma$ in cerebral dissemination of the parasite (Figure 2D).

We then studied the influence of IL-12, a major inducer of IFN- $\gamma$, on parasite migration into the brain parenchyma. At 13 and 20 days p.i., most parasites in brains from IL-12p40-/- mice were found to be intravascular (see Figure 1B). By 29 days p.i.,
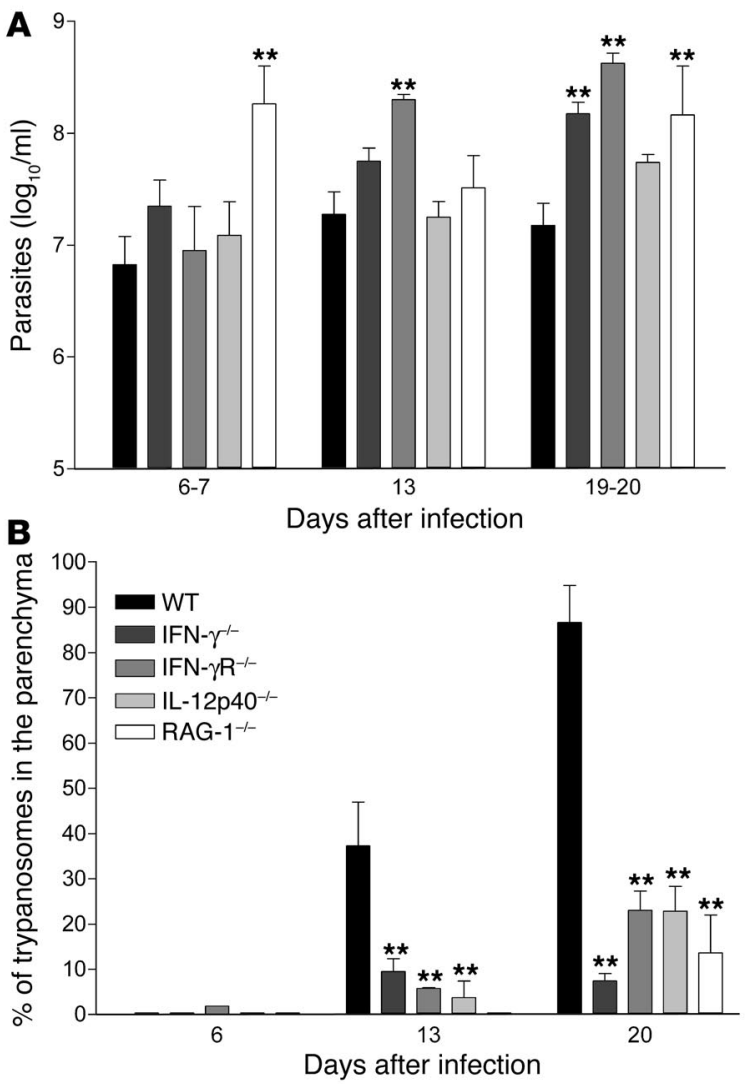

Figure 1

Histograms of parasitemia and trypanosome brain parenchyma invasion. (A) Levels of parasitemia in WT, IFN- $\gamma^{-1-}$, IFN- $\gamma \mathrm{R}^{-1-}$, RAG- $1^{-1-}$, and IL-12p $40^{-/-}$mice at different time points after infection. Each bar represents the mean \pm SEM of the values obtained from 8 animals. (B) Percentage of trypanosomes in the parenchyma of the corpus callosum in the different groups of animals. Each bar represents the mean \pm SEM of the values obtained from 4 animals. The RAG-1-1- brain sections were only examined at 20 days p.i. Statistically significant differences in comparison to WT mice: ${ }^{* *} P<0.01$ (two-way ANOVA followed by Bonferroni test or one-way ANOVA followed by Newman-Keuls multiple comparison test). 


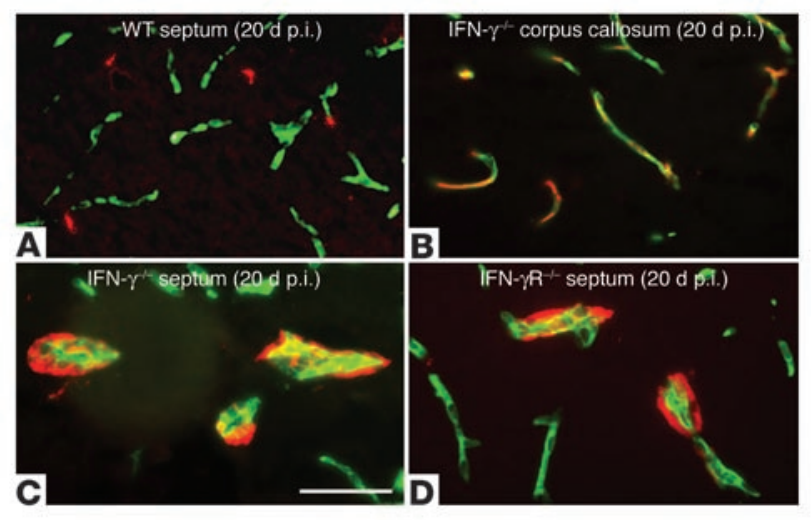

Figure 2

Immunofluorescent images from the septal nuclei and corpus callosum 20 days p.i. (T. brucei brucei in red and endothelial cells in green). (A) In a WT mouse, numerous parasites were observed in the brain parenchyma of the septum. (B) In an IFN- $\gamma^{-1}$ mouse, parasites were localized in the lumen of blood vessels, here illustrated in the corpus callosum. (C) Also in an IFN- $\gamma^{-1-}$ mouse, perivascular aggregates of trypanosomes could be observed in the septal nuclei. (D) In an IFN- $\gamma \mathrm{R}^{--}$mouse, parasites could be found clustered around vessels. Scale bar: $50 \mu \mathrm{m}$.

sporadic clustering of parasites around vessels was detected in IL-12 $440^{-/-}$mice, and the passage of parasites into the parenchyma was significantly reduced.

To study the precise localization of the perivascular aggregates of T. brucei brucei parasites, brain sections from IFN- $\gamma^{-/-}$mice were double-stained with antibodies for T. brucei brucei and for different isoforms of the laminin family of proteins. Immunostaining for the laminin $\beta 1$ and $\gamma 1$ chains, present in both endothelial and parenchymal basement membranes (5), showed that the parasites accumulated in the perivascular space between these two basement membranes (Figures 3 and 4A). The perivascular cuffing of trypanosomes occurred around vessels that were positively stained for the endothelial basement membrane component laminin $\alpha 4$, but had little or no staining for the laminin $\alpha 5$ chain (Figure 4, $\mathrm{B}-\mathrm{D})$. The outer border of the parasite cuffing contained the laminin $\alpha 1$ chain (Figure $4 \mathrm{E}$ ), which is characteristic of the parenchymal basement membrane (5). To investigate whether there were any differences in the endothelial basement membranes of IFN- $\gamma^{-/-}$and WT mice, we stained sections from uninfected and infected mice for laminin $\alpha 4$ and $\alpha 5$ chains. A robust staining in endothelial basement membranes for both laminin chains was observed in whole-brain sections at the level of the lateral ventricles, and there was no apparent difference in the proportion of positively stained blood vessels between the infected and uninfected groups or between IFN- $\gamma^{-/-}$and WT mice.

We then tested whether lymphocytes are a major source of IFN- $\gamma$ in the brains of infected mice and whether they play a role in $T$. brucei brucei penetration into the brain parenchyma. Increased accumulation of IFN- $\gamma$ transcripts was observed in the brains of $T$. brucei brucei-infected WT mice, but not of T. brucei brucei-infected RAG-1/- mice lacking B and T cells (Figure 5). Parasite clustering around vessels in the septal nuclei, hippocampi, and paraventricular thalamic nuclei was observed in brains of $\mathrm{RAG}^{-/-}$mice, similar to that observed in IFN- $\gamma^{-/-}$and IFN- $\gamma \mathrm{R}^{-/-}$mice. Also, the majority of the parasites were still located intravascularly at 19 and 20 days p.i. (see Figure 1B), indicating that IFN- $\gamma$ derived from lymphocytes is necessary for penetration of T. brucei brucei into the brain parenchyma. Moreover, there were reduced numbers of $\mathrm{CD}^{+}$and $\mathrm{CD}^{+}$ cells in the brain parenchyma of the corpus callosum of IFN- $\gamma^{-/-}$, IFN- $\gamma \mathrm{R}^{-/}$, and IL-12 $440^{-/-}$infected mice as compared to WT controls (Figure 6), suggesting that IFN- $\gamma$ is involved in penetration of such cells into the brain parenchyma. In the WT controls, T cells were mainly observed in the parenchyma of the corpus callosum, whereas few $\mathrm{T}$ cells were detected in the cortex and septal nuclei. Thus, IFN- $\gamma$ facilitates penetration of both T. brucei brucei and $\mathrm{T}$ cells from the blood vessels into the brain parenchyma.

\section{Discussion}

This study presents the first systematic effort to define the roles of basement membranes of the cerebral blood vessels and IFN- $\gamma$ in the penetration of T. brucei brucei across the BBB. We demonstrate that laminin isoforms, IFN- $\gamma$, and lymphocytes are involved in determining the infiltration of T. brucei brucei into the brain parenchyma.

T. brucei brucei invasion of the brain was found to be confined to circumventricular organs at 6 days p.i., as described previously in mice and rats (2), while penetration of the parenchyma commenced around 13 days after infection in WT mice. Similar to our observations in rats (3), parasite invasion occurred by the penetration of intracerebral vessels and not by the spread of parasites from the circumventricular organs through the cerebrospinal fluid, since few parasites were observed close to the ventricles. Furthermore, epithelial cells, including the choroid plexus epithelial cells, express the laminin $\alpha 5$ chain, which we have shown to be nonpermissive for $T$. brucei brucei transmigration out of cerebral vessels (23).

Similar to the situation in murine toxoplasmic encephalitis (24), where leukocyte recruitment into the brain parenchyma is reduced in the absence of IFN- $\gamma$, we found that the penetration of $\mathrm{T}$ cells across the BBB was reduced in IFN- $\gamma^{-/-}$compared to WT mice. A most remarkable finding in our study was that the penetration across the BBB of both $\mathrm{T}$ cells and T. brucei brucei was impeded in the IFN- $\gamma^{-/-}$mice, although these mice displayed higher parasitemia than WT controls. Invasion of T. brucei brucei was also inhibited in RAG-1/- mice, suggesting that B and/or T cells facilitate migration of T. brucei brucei into the brain and that innate immune cell-derived IFN- $\gamma$ is not sufficient for T. brucei

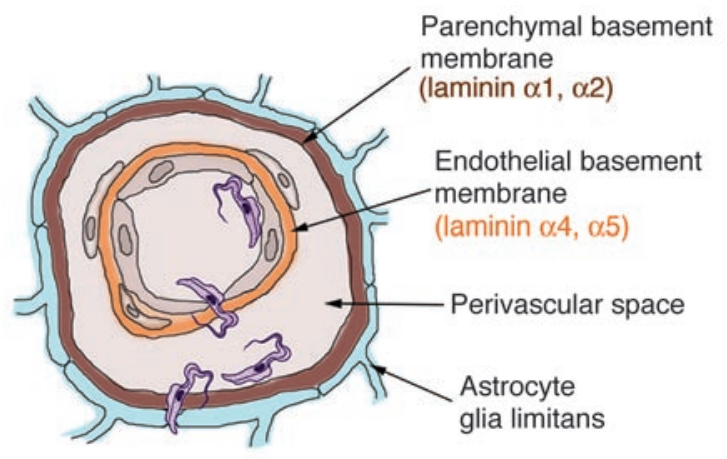

\section{Figure 3}

Schematic drawing of a cerebral vessel showing passage of trypanosomes (purple) across the two basement membranes. Localization of the laminin $\alpha 1$ and $\alpha 2$ chains in the parenchymal basement membrane and that of $\alpha 4$ and $\alpha 5$ chains in the endothelial basement membrane are shown as well. 

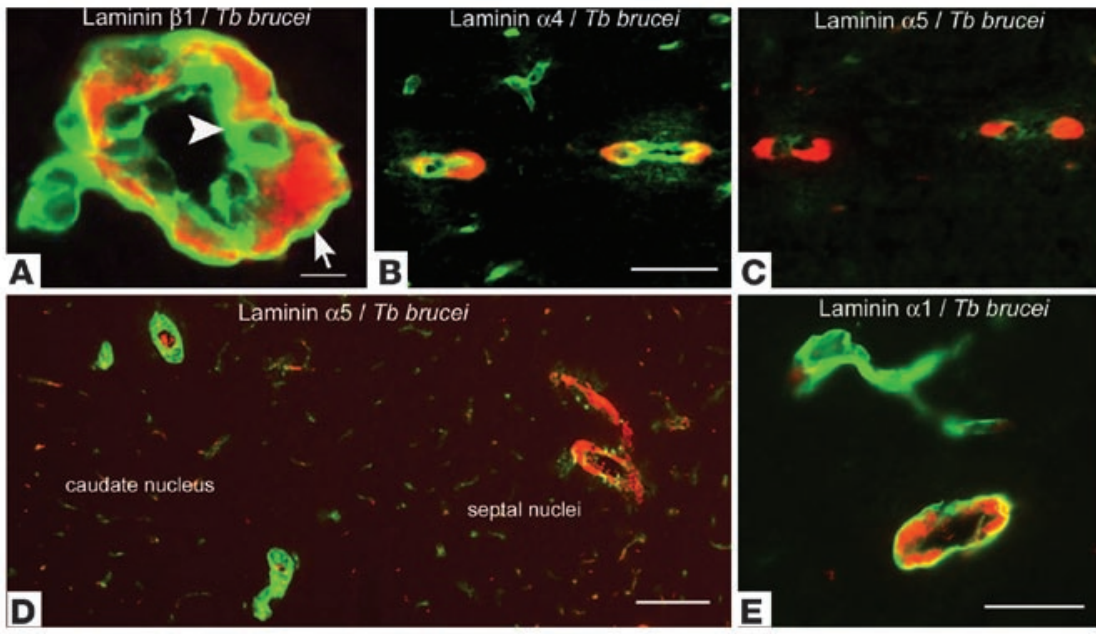

\section{Figure 4}

Immunofluorescent images from the septal nuclei of IFN- $\gamma^{-1-}$ mice, with double immunostaining of $T$. brucei brucei (red) and different laminin isoforms (green). (A) The parasites were clustered and confined between the endothelial (arrowhead) and parenchymal (arrow) basement membranes stained with anti-laminin $\beta 1$. (B-D) Parasites clustered around vessels containing the endothelial laminin isoform $\alpha 4$ but not around those containing laminin $\alpha 5$ (B and $\mathbf{C}$ are adjacent sections). Note that in $\mathbf{D}$, laminin $\alpha 5$ occurs in association with vessels in the caudate nuclei but not vessels in the septal nuclei with accumulation of parasites around them. (E) The parasites accumulate inside the parenchymal basement membranes stained with anti-laminin $\alpha 1$. Tb, Trypanosoma brucei. Scale bars: A, $10 \mu \mathrm{m}$; B-E, $50 \mu \mathrm{m}$. brucei penetration through the BBB. IFN- $\gamma$ may have direct effects on T. brucei brucei $(25,26)$. However, a similar pattern of T. brucei brucei neuroinvasion was observed in IFN- $\gamma^{-/-}$and IFN- $\gamma \mathrm{R}^{-/-}$mice, indicating that IFN- $\gamma$ facilitates T. brucei brucei migration into the brain parenchyma by acting on the tissues of the host rather than by altering the phenotype of the parasites.

Interestingly, a marked accumulation of parasites surrounding the cerebral endothelial cells was observed in the septal nuclei, hippocampi, and paraventricular thalamic nuclei of IFN- $\gamma^{-/-}$, IFN- $\gamma \mathrm{R}^{-/}$, and RAG-1/- mice. At early stages of murine EAE, lymphocytes accumulate between the two distinct basement membranes surrounding cerebral vessels - the endothelial and parenchymal basement membranes - which contain different laminin isoforms defined by specific laminin $\alpha$ chains (5). Endothelial basement membranes containing laminin $\alpha 4$ are permissive for $T$ cell transmigration, while those containing laminin $\alpha 5$ are not (5). In the present study, although parasites managed to cross the tight junctions and the endothelial basement membrane of the post-capillary venules in the septal nuclei, hippocampi, and paraventricular thalamic nuclei, they could not traverse the outer parenchymal basement membrane that acts as the border to the brain parenchyma. Since T. brucei brucei clustering was observed around laminin $\alpha 4$-containing vessels, but not around vessels showing robust laminin $\alpha 5$ immunostaining, T. brucei brucei may utilize mechanisms similarly to lymphocytes to invade the nervous system. Alternatively, lymphocytes could alter the structural integrity of the basement membranes in an IFN- $\gamma$-mediated way, allowing subsequent penetration by parasites.

IFN- $\gamma$ can induce macrophage- and microglial cell-derived TNF- $\alpha$ and nitric oxide secretion (10). In turn, TNF- $\alpha$ and NO activate matrix metalloproteinases $(27,28)$ that selectively cleave basement membrane components to facilitate migration of leukocytes into the brain (29-32). A similar mechanism may operate for migration of T. brucei brucei across the BBB. In support of this hypothesis, it has been reported that IFN- $\gamma^{-/-}$mice express low levels of $\mathrm{NO}$ and TNF- $\alpha(18,33)$. In addition, IFN- $\gamma$ can induce chemokines (in astrocytes, macrophages, and microglial cells), which are involved in leukocyte recruitment into the brain $(24,34)$.

In conclusion, we have shown that, similar to its role in lymphocyte invasion of the nervous system, the composition of the basement membranes of the intracerebral ves- sels plays a decisive role in determining the sites of T. brucei brucei penetration into the brain. We also demonstrated that the parasite penetration through the parenchymal basement membrane can be facilitated by IFN- $\gamma$. IFN- $\gamma$ can thus be involved, on the one hand, in stimulating the immunological control of the infection, while on the other hand, it can have a paradoxical effect of promoting T. brucei brucei penetration through the BBB and invasion of the brain parenchyma. These findings could be of relevance for a better staging of the disease through identification of biomarkers induced by IFN- $\gamma$ during $T$. brucei brucei penetration into the brain and also for development of treatment strategies aimed at inhibiting parasite penetration across the BBB, which would supplement the slow-acting drugs that are currently used to clear the systemic infection.

\section{Methods}

Mice, parasites, and infection. Mice deficient in IFN- $\gamma(35), \operatorname{IFN}-\gamma \mathrm{R}(36)$, IL-12p40 (37), or RAG-1 (38) were generated by homologous recombination in embryonic stem cells. All mouse strains were back-crossed for 9 generations with $\mathrm{C} 57 \mathrm{BL} / 6$, and $\mathrm{C} 57 \mathrm{BL} / 6$ mice were used as controls.

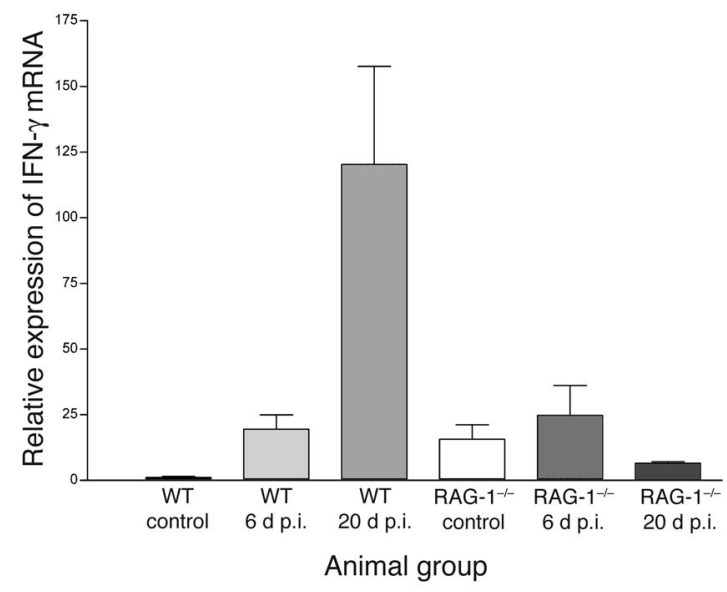

Figure 5

Histogram showing the relative expression of IFN- $\gamma$ mRNA in the brains of WT and RAG-1-1- uninfected mice and infected mice. Each bar represents the mean \pm SEM of the values obtained from 4 animals. 


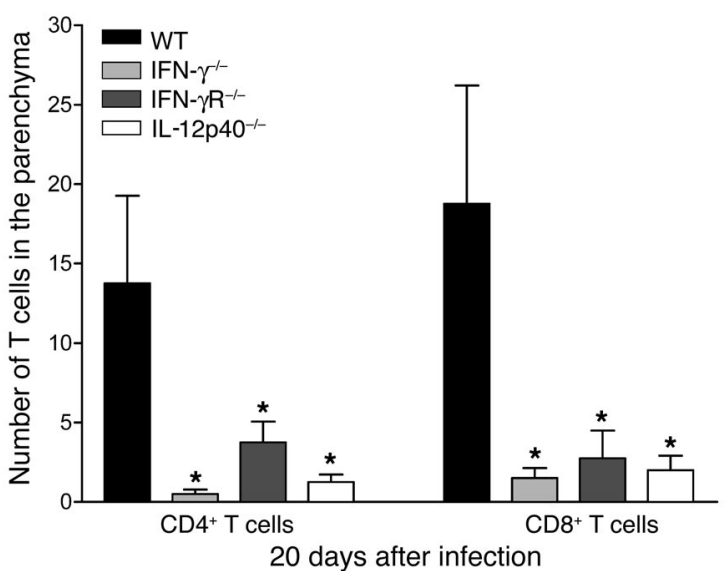

Figure 6

Histogram showing the numbers of $\mathrm{CD}^{+}$and $\mathrm{CD} 8^{+} \mathrm{T}$ cells in the parenchyma of the corpus callosum of the different groups of animals (WT, IFN- $\gamma^{-1-}$, IFN- $\gamma \mathrm{R}^{-1-}$, and IL-12p $40^{-/-}$mice) at 20 days p.i. Each bar represents the mean \pm SEM of the values obtained from 4 animals. Statistically significant differences in comparison to B6 WT mice: ${ }^{*} P<0.05$ (oneway ANOVA followed by Newman-Keuls multiple comparison test).

All experiments were conducted following protocols that received institutional approval and authorization by the local animal ethical committee (Stockholms Norra Djurförsöksetiska Nämnd, project N362/02). Efforts were made to minimize the numbers and suffering of animals used. Mice were supplied by the breeding unit at the Microbiology and Tumour Biology Center, Karolinska Institutet, Sweden, and were kept with food and water ad libitum under specific pathogen-free conditions.

Mice (8-12 weeks old) were infected by intraperitoneal injection with 2,000 parasites of a pleomorphic stabilate of T. brucei brucei, AnTat 1:1E, derived from stabilate EATRO $1125(1 \times 3$ day passage in CB57 mice; obtained from N. van Meirveinne, Laboratory of Serology, Institute of Tropical Medicine "Prince Leopold", Antwerp, Belgium). Parasites were diluted in $60 \mathrm{mM}$ PBS containing $40 \mathrm{mM}$ glucose. Animals were checked and weighed daily for signs of disease. Blood samples were taken from the tip of the tail during the course of infection to assess parasitemia using the Herbet and Lumsden chart (39).

Immunobistochemical technique. Brains were dissected at different times after infection, and brain sections were analyzed by immunohistochemistry. Briefly, frontal serial sections (14 $\mu \mathrm{m}$ thick) were cut on a cryostat and thaw-mounted on chrome-alum gelatincoated slides. Sections were collected from the level of the lateral ventricles containing the choroid plexus and the septal nuclei. In addition, brains from IFN- $\gamma^{-/-}$, IFN- $\gamma \mathrm{R}^{-/}$, and RAG-1 $1^{-/}$mice were examined at different levels throughout the rostrocaudal axis of the brain. Prior to immunohistochemical processing, the sections were fixed in $4 \%$ formalin and $0.17 \%$ picric acid in PBS for 30 seconds at $4{ }^{\circ} \mathrm{C}$, rinsed in PBS, fixed in acetone for 30 seconds at $-20^{\circ} \mathrm{C}$, and then rinsed in PBS. All sections were preincubated with $1 \%$ bovine serum albumin and $0.3 \%$ Triton X-100 in PBS (all primary and secondary antisera were diluted in this solution) for 1 hour at room temperature. One section per slide, from each animal, was then incubated with a mixture of rabbit polyclonal anti-variant surface glycoprotein (anti-VSG) of the AnTat 1:1E stabilate $(1: 5,000)$ and goat polyclonal anti-GLUT-1 (1:40; Santa Cruz Biotechnology) antisera for 24-48 hours at $4^{\circ} \mathrm{C}$. Sections were then rinsed in PBS and incubated with a mixture of donkey anti-rabbit IgG conjugated to Rhodamine Red (1:200; Jackson Immuno Research) and donkey anti-goat IgG conjugated to
Alexa 488 (1:100; Invitrogen Corp.) for 60 minutes at room temperature. The sections were rinsed and mounted in glycerol mixed with $2.5 \%$ diazabicyclanooctane (Sigma-Aldrich). Additional sections from the IFN- $\gamma^{-/-}$ and IFN- $\gamma \mathrm{R}^{-/-}$mice were incubated with a mixture of anti-VSG and either monoclonal rat anti-mouse laminin $\beta 1$ (1:1; clone 3A4) (5) or rat antimouse laminin $\gamma 1$ (supernatant; clone 3E10) (5). Alternating sections from IFN- $\gamma^{-/-}$mice were further incubated with a mixture of anti-VSG and rat anti-mouse laminin $\alpha 4$ (1:300; serum 341) (40), rat anti-mouse laminin $\alpha 5$ (supernatant; clone 4G6) (41), or rat anti-mouse laminin $\alpha 1$ (1:500; clone 198) (42). Sections were then processed as described above and incubated with a mixture of donkey anti-rabbit IgG conjugated to Rhodamine Red and donkey anti-rat IgG conjugated to Cy2 (1:100; Jackson Immuno Labs). Another set of sections from uninfected and infected WT and IFN- $\gamma^{-/-}$mice were incubated with rat anti-mouse laminin $\alpha 4$ and $\alpha 5$. Donkey anti-rat IgG conjugated to Rhodamine Red was used as a secondary antibody.

To examine the migration of leukocytes into the brain parenchyma, sections from WT, IFN- $\gamma^{-/-}$, IFN- $\gamma \mathrm{R}^{-/-}$, and IL-12 $40^{-/-}$mice were incubated with a mixture of goat anti-GLUT-1 combined with either rat antimouse CD4 $4^{+}$(1:200; BD Biosciences - Pharmingen) or CD8 ${ }^{+}$(1:200; BD Biosciences - Pharmingen). As secondary antibodies, a mixture of donkey anti-goat IgG conjugated to Cy2 and donkey anti-rat IgG (adsorbed against mouse $\operatorname{IgG}$ ) conjugated to Rhodamine Red was used.

Sections were examined and analyzed using a Nikon Corp. fluorescence microscope. Photomicrographs were taken with a Carl Zeiss Vision AxioCam digital camera.

Quantitative evaluations. We counted the number of VSG-immunopositive T. brucei brucei in 5 ocular fields (viewed through a $\times 10$ ocular, $\times 20$ objective) from the cortex and corpus callosum on either side of the midline, as well as in 4 fields from the septal nuclei in 4 animals from each p.i. time point. The parasites were divided into two groups according to their relationship with the vessels: intravascular or extravascular. $\mathrm{CD} 4^{+}$and $\mathrm{CD}^{+} \mathrm{T}$ cells were counted in a similar manner to that described for the parasites.

Real-time RT-PCR. IFN- $\gamma$ and cyclophilin transcripts were quantified in brains from WT and RAG-1/- uninfected and infected mice by real-time RT-PCR. Total RNA was extracted from half of the fresh frozen brains using the RNeasy Kit (Qiagen GmbH). Subsequently, $500 \mathrm{ng}$ of total RNA was treated with $1 \mathrm{U}$ DNase I (Invitrogen Corp.) for 15 minutes at room temperature and inactivated by the addition of $2.5 \mathrm{mM}$ EDTA followed by incubation at $65^{\circ} \mathrm{C}$ for 10 minutes. cDNA synthesis of 500 ng RNA was completed in a $40-\mu \mathrm{l}$ reaction containing $100 \mathrm{U}$ of Superscript II RNse $\mathrm{H}^{-}$reverse transcriptase, $150 \mathrm{ng}$ of random hexenucleotides, first strand buffer (1:5), $10 \mathrm{mM}$ DTT, and $0.5 \mathrm{mM} \mathrm{dNTPs} \mathrm{(all} \mathrm{from} \mathrm{Invitrogen} \mathrm{Corp.)}$ for 1 hour at $42^{\circ} \mathrm{C}$ before inactivation at $70^{\circ} \mathrm{C}$ for 15 minutes.

The real-time PCR was performed in three $25-\mu \mathrm{l}$ reactions containing Platinum SYBR Green qPCR Supermix-UDG (1:2; Invitrogen Corp.), 125 $\mathrm{nM}$ of forward and reverse primers, and $1 \mu \mathrm{l}$ of cDNA on an ABI Prism 7000 sequence detection system (Applied Biosystems). The following primer sequences were used: sense IFN- $\gamma, 5^{\prime}$-ACAATGAACGCTACACACTGCAT- $3^{\prime}$; antisense IFN- $\gamma, 5^{\prime}$-TGGCAGTAACAGCCAGAAACA3'; sense cyclophilin, 5'-GCTTTTCGCCGCTTGCT-3'; and antisense cyclophilin, 5'-CTCGTCATCGGCCGTGAT-3'. 10-fold dilutions of a cDNA sample were amplified to control amplification efficiency for each primer pair. Thereafter, the $C_{t}$ values for all cDNA samples were obtained. The amount of transcripts of individual samples $(n=4$ per group) was normalized to cyclophilin $\left(\Delta C_{t}\right)$. The relative amount of IFN- $\gamma$ transcripts was calculated using the $2^{-\Delta \Delta C_{t}}$ method as previously described (43). These values were then used to calculate the mean and standard error of the relative expression of IFN- $\gamma$ mRNA in the brain of uninfected and infected mice. 


\section{Acknowledgments}

This study was supported by grants from the United Nations Development Programme (UNDP)/World Bank/WHO Special Programme for Research and Training in Tropical Diseases (TDR), the Swedish Research Council (4480), and the National Heart, Lung, and Blood Institute (1-R01-H1-71510-01). Karolina Kristensson is gratefully acknowledged for drawing Figure 3.
Received for publication May 7, 2004, and accepted in revised form July 6, 2004.

Address correspondence to: Krister Kristensson, Department of Neuroscience, Retzius väg 8, Karolinska Institutet, SE-171 77 Stockholm, Sweden. Phone: 46-8-728-78-25; Fax: 46-8-32-53-25; E-mail: krister.kristensson@neuro.ki.se.
1. Dumas, M., and Bisser, S. 1999. Clinical aspects of human African trypanosomiasis. In Progress in human African trypanosomiasis, sleeping sickness. M. Dumas, B. Bouteille, and A. Buguet, editors. Springer. Paris, France. 215-233.

2. Schultzberg, M., Ambatsis, M., Samuelsson, E.B., Kristensson, K., and van Meirvenne, N. 1988 Spread of Trypanosoma brucei to the nervous system: early attack on circumventricular organs and sensory ganglia. J. Neurosci. Res. 21:56-61.

3. Mulenga, C., Mhlanga, J.D., Kristensson, K., and Robertson, B. 2001. Trypanosoma brucei brucei crosses the blood-brain barrier while tight junction proteins are preserved in a rat chronic disease model. Neuropathol. Appl. Neurobiol. 27:77-85.

4. Rubin, L.L., and Staddon, J.M. 1999. The cell biology of the blood-brain barrier. Annu. Rev. Neurosci. 22:11-28.

5. Sixt, M., et al. 2001. Endothelial cell laminin isoforms, laminins 8 and 10, play decisive roles in $\mathrm{T}$ cell recruitment across the blood-brain barrier in experimental autoimmune encephalomyelitis. J. Cell Biol. 153:933-946.

6. Aumailley, M., and Smyth, N. 1998. The role of laminins in basement membranes. J. Anat. 193:1-21.

7. Timpl, R., and Brown, J. 1994. The laminins. Matrix Biol. 14:275-281.

8. Tunggal, P., Smyth, N., Paulsson, M., and Ott, M.C. 2000. Laminins: structure and genetic regulation. Micro. Res. Tech. 51:214-227.

9. Gloor, S.M., et al. 2001. Molecular and cellular permeability control at the blood-brain barrier. Brain Res. Brain Res. Rev. 36:258-264.

10. Owens, T., et al. 2001. Pathogenesis of encephalitis. In Neuroimmune biology. Vol. 1: New foundation of biology. I. Berczi and M. Gorczynski, editors. Elsevier. Amsterdam, The Netherlands. 387-397.

11. Eugenin, E.A., and Berman, J.W. 2003. Chemokinedependent mechanisms of leukocyte trafficking across a model of the blood-brain barrier. Methods. 29:351-361.

12. Boehm, U., Klamp, T., Groot, M., and Howard, J.C. 1997. Cellular responses to interferon-gamma. Annu. Rev. Immunol. 15:749-795.

13. Ma, X., et al. 1996. The interleukin 12 p40 gene promoter is primed by interferon gamma in monocytic cells. J. Exp. Med. 183:147-157.

14. Wenner, C.A., Guler, M.L., Macatonia, S.E., O'Garra, A., and Murphy, K.M. 1996. Roles of IFNgamma and IFN-alpha in IL-12-induced T helper cell-1 development. J. Immunol. 156:1442-1447.

15. Smeltz, R.B., Chen, J., Ehrhardt, R., and Shevach, E.M. 2002. Role of IFN-gamma in Th1 differentiation: IFN-gamma regulates IL-18R alpha expression by preventing the negative effects of IL-4 and by inducing/maintaining IL-12 receptor beta 2 expression. J. Immunol. 168:6165-6172.
16. Szabo, S.J., Dighe, A.S., Gubler, U., and Murphy, K.M. 1997. Regulation of the interleukin (IL)-12R beta 2 subunit expression in developing $\mathrm{T}$ helper 1 (Th1) and Th2 cells. J. Exp. Med. 185:817-824.

17. Hertz, C.J., Filutowicz, H., and Mansfield, J.M. 1998 Resistance to the African trypanosomes is IFNgamma dependent. J. Immunol. 161:6775-6783.

18. Namangala, B., Noel, W., De Baetselier, P., Brys, L., and Beschin, A. 2001. Relative contribution of interferon-gamma and interleukin-10 to resistance to murine African trypanosomosis. J. Infect. Dis. 183:1794-1800.

19. Darji, A., Sileghem, M., Heremans, H., Brys, L., and De Baetselier, P. 1993. Inhibition of T-cell responsiveness during experimental infections with Trypanosoma brucei: active involvement of endogenous gamma interferon. Infect. Immun. 61:3098-3102.

20. Shi, M., Pan, W., and Tabel, H. 2003. Experimental African trypanosomiasis: IFN-gamma mediates early mortality. Eur. J. Immunol. 33:108-118.

21. Uzonna, J.E., Kaushik, R.S., Gordon, J.R., and Tabel, H. 1998. Experimental murine Trypanosoma congolense infections. I. Administration of anti-IFN gamma antibodies alters trypanosome-susceptible mice to a resistant-like phenotype. J. Immunol. 161:5507-5515.

22. Olsson, T., et al. 1993. CD8 is critically involved in lymphocyte activation by a T. brucei brucei-released molecule. Cell. 72:715-727.

23. Sorokin, L.M., Pausch, F., Durbeej, M., and Ekblom, P. 1997. Differential expression of five laminin $\alpha(1-5)$ chains in developing and adult mouse kidney. Dev. Dyn. 210:446-462.

24. Strack, A., Asensio, V.C., Campbell, I.L., Schluter, D., and Deckert, M. 2002. Chemokines are differentially expressed by astrocytes, microglia and inflammatory leukocytes in Toxoplasma encephalitis and critically regulated by interferon-gamma. Acta Neuropathol. (Berl.). 103:458-468.

25. Olsson, T., et al. 1991. Bidirectional activating signals between Trypanosoma brucei and CD8+ T cells: a trypanosome-released factor triggers interferon-gamma production that stimulates parasite growth. Eur. J. Immunol. 21:2447-2454.

26. Hua, S.B., and Wang, C.C. 1997. Interferon-gamma activation of a mitogen-activated protein kinase, KFR1, in the bloodstream form of Trypanosoma brucei.J. Biol. Chem. 272:10797-10803.

27. Rosenberg, G.A. 1995. Matrix metalloproteinases in brain injury. J. Neurotrauma. 12:833-842.

28. Trachtman, H., Futterweit, S., and Singhal, P. 1995. Nitric oxide modulates the synthesis of extracellular matrix proteins in cultured rat mesangial cells. Biochem Biophys. Res. Commun. 207:120-125.

29. Rosenberg, G.A., et al. 1992. TIMP-2 reduces proteolytic opening of blood-brain barrier by type IV collagenase. Brain Res. 576:203-207.

30. Cuzner, M.L., and Opdenakker, G. 1999. Plasminogen activators and matrix metalloproteases, mediators of extracellular proteolysis in inflammatory demyelination of the central nervous system. J. Neuroimmunol. 94:1-14.

31. Graesser, D., Mahooti, S., and Madri, J.A. 2000. Distinct roles for matrix metalloproteinase-2 and alpha4 integrin in autoimmune $T$ cell extravasation and residency in brain parenchyma during experimental autoimmune encephalomyelitis. J. Neuroimmunol. 109:121-131.

32. Hartung, H.P., and Kieseier, B.C. 2000. The role of matrix metalloproteinases in autoimmune damage to the central and peripheral nervous system. J. Neuroimmunol. 107:140-147.

33. Hertz, C.J., and Mansfield, J.M. 1999. IFN-gammadependent nitric oxide production is not linked to resistance in experimental African trypanosomiasis. Cell Immunol. 192:24-32.

34. Tran, E.H., Prince, E.N., and Owens, T. 2000. IFNgamma shapes immune invasion of the central nervous system via regulation of chemokines. J. Immunol. 164:2759-2768.

35. Dalton, D.K., et al. 1993. Multiple defects of immune cell function in mice with disrupted interferon-gamma genes. Science. 259:1739-1742.

36. Huang, S., et al. 1993. Immune response in mice that lack the interferon-gamma receptor. Science. 259:1742-1745.

37. Magram, J., et al. 1996. IL-12-deficient mice are defective in IFN gamma production and type 1 cytokine responses. Immunity. 4:471-481.

38. Mombaerts, P., et al. 1992. RAG-1-deficient mice have no mature $\mathrm{B}$ and $\mathrm{T}$ lymphocytes. Cell. 68:869-877.

39. Herbert, W.J., and Lumsden, W.H. 1976. Trypanosoma brucei: a rapid "matching" method for estimating the host's parasitemia. Exp. Parasitol. 40:427-431.

40. Ringelmann, B., et al. 1999. Expression of laminin $\alpha 1, \alpha 2, \alpha 4$ and $\alpha 5$ chains, fibronectin and tenascin$\mathrm{C}$ in skeletal muscle of dystrophic 129ReJ $d y / d y$ mice. Exp. Cell Res. 246:165-182.

41. Sorokin, L.M., et al. 1997. Developmental regulation of the laminin $\alpha 5$ chain suggests a role in epithelial and endothelial cell maturation. Dev. Biol. 189:285-300.

42. Sorokin, L.M., et al. 1992. Monoclonal antibodies against the laminin A chain fragment E3 and their effects on cell- and heparin-binding and on kidney development. Exp. Cell Res. 201:137-144.

43. Livak, K.J., and Schmittgen, T.D. 2001. Analysis of relative gene expression data using real-time quantitative PCR and the 2(-Delta Delta C(T)) Method. Methods. 25:402-408. 